\title{
New Method of Measuring the Positive-sequence Capacitance of T-connection Transmission Lines
}

\author{
Yuansen Zhang ${ }^{1}$, Demin Cui ${ }^{1}$, Qingtao Cao ${ }^{1}$, Yongqiang Chai ${ }^{1}$, Peng Liu ${ }^{1}$, Xiaobo Li ${ }^{1}$, \\ Zhijian $\mathrm{Hu}^{2}$, Chuanqi $\mathrm{Li}^{2}$, Chengxue Zhang ${ }^{2}$ \\ ${ }^{1}$ Dezhou Power Supply Company, Shandong Electric Power Group Co., Dezhou, China \\ ${ }^{2}$ School of Electrical Engineering, Wuhan University, Wuhan, China \\ Email: zhijian_hu@163.com
}

Received February, 2013

\begin{abstract}
A novel method of measuring the positive-sequence capacitance of T-connection transmission lines is proposed. The mathematical model of the new method is explained in detail. In order to obtain enough independent equations, three independent operation modes of T-connection transmission lines during the line measurement are introduced. The digital simulation results and field measurement results are shown. The simulation and measurement results have validated that the new method can meet the needs of measuring the positive-sequence capacitance of T-connection transmission lines. This method has been implemented in the newly developed measurement instrument.
\end{abstract}

Keywords: T-connection Transmission Lines; Line Parameter; Positive-sequence Capacitance; Measurement

\section{Introduction}

With the rapid development of power systems, line corridors become more and more crowed. In order to reduce synthetic cost of the line construction and limited objective conditions, T-connection transmission lines are always applied in HV/EHV. The reliable operation of power systems depends on the correct operation of relaying protection devices [1].

It is well known that the computation for parameters of T-connection transmission lines is derived from Carson formula [2]. It is influenced by many practical factors, such as the resistance of earth, the equivalent depth of the lines and, etc. So the parameters should be practically measured rather than theoretically calculated.

There are many related literatures discussing about measuring the impedance parameters of T-connection transmission lines [3-5]. The capacitance parameters are always ignored. But the capacitance is very important for transmission line protection. When it exceeds certain length, because the numerical value of capacity current is big, the current flow on both sides of the lines and phase relation will change according with the increase of capacity current value. Especially, when load current and short-circuit current are small, it can lead to the maloperation of high frequency protection [6, 7]. If we use the traditional method to measure the capacitance of T-connection transmission lines, it can only calculate parallel values of three branches. When the parameters of three branches are different, the traditional method no longer works. So, new measurement method needs to be found out for the positive-sequence capacitance of T-connection transmission lines.

In this paper, a new method of measuring positivesequence capacitance of T-connection lines is proposed. The method has been applied to the newly developed measurement instrument.

\section{The Theory of the Measurement Method}

Usually, the length of T-connection transmission lines is less than $50 \mathrm{~km}$. So it can use the lumped parameter model. Each branch can be equivalent to T-form circuit. The lumped parameter model of T-form equivalent circuit is shown in Figure 1.

When the length and impedance of transmission lines are known, the susceptance can be calculated. Three phases end is opening circuit, $\dot{I}_{2}=0$, the susceptance $y$ is as follows,

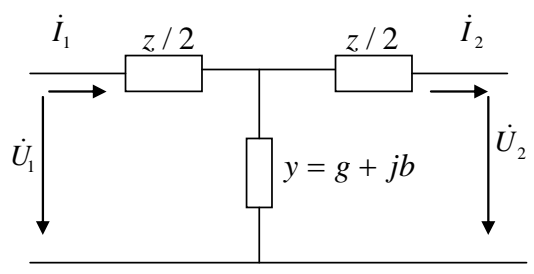

Figure 1. The lumped parameter model of T-form equivalent circuit. 


$$
y=\frac{1}{\left(\dot{U}_{1} / \dot{I}_{1}\right)-(z / 2)}=g+j b
$$

where, $\dot{U}_{1}$ is the positive-sequence voltage vector at the head of the line; $\dot{I}_{1}$ is the positive-sequence current vector at the head of the line.

Then, the capacitance can be written as follows,

$$
c=\frac{b}{L w}
$$

where, $L$ is the length of the transmission lines; w equals to $2 \pi f$ and $f$ is power frequency.

Figure 1 can be applied to three branches of T-connection transmission lines. The equivalent model is shown in Figure 2.

The impedance parameters of three branches are known. In order to obtain enough independent equations, three independent operation modes of $\mathrm{T}$-connection transmission lines during the measurement are shown in Table 1. In order to simplify the equations, substitute half of the impedance parameters of three branches with $\mathrm{Z}_{1}, \mathrm{Z}_{2}$ and $\mathrm{Z}_{3}$. Then, we have,

$$
\left\{\begin{array}{l}
\frac{\dot{U}_{11}}{\dot{I}_{11}}=\frac{\left[\frac{\left(Z_{2}+Y_{2}\right)\left(Z_{3}+Y_{3}\right)}{Z_{2}+Y_{2}+Z_{3}+Y_{3}}+Z_{1}\right] \times Y_{1}}{\left[\frac{\left(Z_{2}+Y_{2}\right)\left(Z_{3}+Y_{3}\right)}{Z_{2}+Y_{2}+Z_{3}+Y_{3}}+Z_{1}\right]+Y_{1}}+Z_{1} \\
\frac{\dot{U}_{22}}{\dot{I}_{22}}=\frac{\left[\frac{\left(Z_{1}+Y_{1}\right)\left(Z_{3}+Y_{3}\right)}{Z_{1}+Y_{1}+Z_{3}+Y_{3}}+Z_{2}\right] \times Y_{2}}{\left[\frac{\left(Z_{1}+Y_{1}\right)\left(Z_{3}+Y_{3}\right)}{Z_{1}+Y_{1}+Z_{3}+Y_{3}}+Z_{2}\right]+Y_{2}}+Z_{2} \\
\left.\frac{\left[\frac{\left(Z_{1}+Y_{1}\right)\left(Z_{2}+Y_{2}\right)}{Z_{1}+Y_{1}+Z_{2}+Y_{2}}+Z_{3}\right] \times Y_{3}}{\dot{U}_{33}}=\frac{\left(Z_{1}+Y_{1}\right)\left(Z_{2}+Y_{2}\right)}{\dot{I}_{33}+Y_{1}+Z_{2}+Y_{2}}+Z_{3}\right]+Y_{3}
\end{array}\right.
$$

Equation (3) can be simplified as,

$$
\left\{\begin{array}{l}
\frac{\dot{U}_{11}}{\dot{I}_{11}}=Z_{1}+\frac{\left(Z_{1} Z_{2}+Z_{2} Z_{3}+Z_{1} Z_{3}\right) Y_{1}+\left(Z_{1}+Z_{2}\right) Y_{1} Y_{3}+\left(Z_{1}+Z_{3}\right) Y_{1} Y_{2}+Y_{1} Y_{2} Y_{3}}{\left(Z_{1} Z_{2}+Z_{2} Z_{3}+Z_{1} Z_{3}\right) Y_{1}+\left(Z_{1}+Z_{2}\right) Y_{3}+\left(Z_{1}+Z_{3}\right) Y_{2}+\left(Z_{2}+Z_{3}\right) Y_{1}+Y_{1} Y_{2}+Y_{2} Y_{3}+Y_{1} Y_{3}} \\
\frac{\dot{U}_{22}}{\dot{I}_{22}}=Z_{2}+\frac{\left(Z_{1} Z_{2}+Z_{2} Z_{3}+Z_{1} Z_{3}\right) Y_{2}+\left(Z_{2}+Z_{3}\right) Y_{1} Y_{2}+\left(Z_{1}+Z_{2}\right) Y_{2} Y_{3}+Y_{1} Y_{2} Y_{3}}{\left(Z_{1} Z_{2}+Z_{2} Z_{3}+Z_{1} Z_{3}\right) Y_{1}+\left(Z_{1}+Z_{2}\right) Y_{3}+\left(Z_{1}+Z_{3}\right) Y_{2}+\left(Z_{2}+Z_{3}\right) Y_{1}+Y_{1} Y_{2}+Y_{2} Y_{3}+Y_{1} Y_{3}} \\
\frac{\dot{U}_{33}}{\dot{I}_{33}}=Z_{3}+\frac{\left.\left(Z_{1} Z_{2}+Z_{2} Z_{3}+Z_{1} Z_{3}\right) Y_{3}+\left(Z_{1}+Z_{3}\right) Y_{2} Y_{3}+\left(Z_{2}+Z_{3}\right) Y_{1} Y_{3}\right)+Y_{1} Y_{2} Y_{3}}{\left(Z_{1} Z_{2}+Z_{2} Z_{3}+Z_{1} Z_{3}\right) Y_{1}+\left(Z_{1}+Z_{2}\right) Y_{3}+\left(Z_{1}+Z_{3}\right) Y_{2}+\left(Z_{2}+Z_{3}\right) Y_{1}+Y_{1} Y_{2}+Y_{2} Y_{3}+Y_{1} Y_{3}}
\end{array}\right.
$$

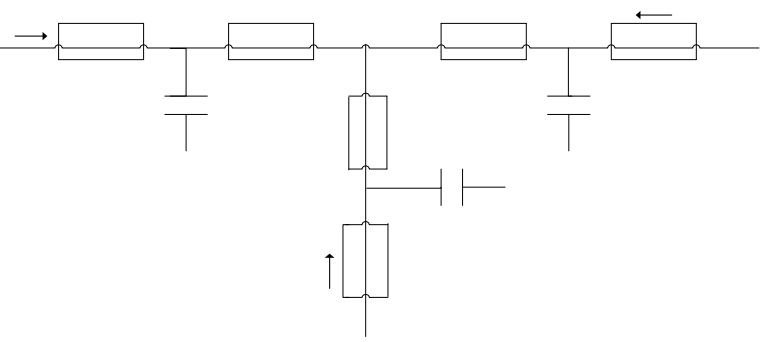

Figure 2. The equivalent model of T-connect transmission lines.

Table 1. The Measurement Modes of Three Braches.

\begin{tabular}{clll}
\hline Cases & \multicolumn{1}{c}{ Branch 1 } & \multicolumn{1}{c}{ Branch 2 } & Branch 3 \\
\hline \multirow{2}{*}{1} & $\begin{array}{l}\text { Applied with an external positive-sequence } \\
\text { voltage source }\end{array}$ & Open circuit & Open circuit \\
2 & Open circuit & $\begin{array}{l}\text { Applied with an external positive-sequence } \\
\text { voltage source }\end{array}$ & Open circuit \\
3 & Open circuit & Open circuit & $\begin{array}{l}\text { Applied with an external positive-sequence } \\
\text { voltage source }\end{array}$ \\
\hline
\end{tabular}


Where $Z_{1}, Z_{2}$ and $Z_{3}$ are half of the positive-sequence impedance parameters of three branches; $\mathrm{Y}_{1}, \mathrm{Y}_{\underline{2}}$ and $\mathrm{Y}_{\underline{3}}$ are the reciprocal of the positive-sequence susceptance parameters of three branches; $\dot{U}_{11}, \dot{U}_{22}$ and $\dot{U}_{33}$ are the positive-sequence voltage vectors of three branches; $\dot{I}_{11}, \dot{I}_{22}$ and $\dot{I}_{33}$ are the positive-sequence current vectors of three branches; Subscripts 11, 22 and 33 mean that the first number represents one of the three branches and the second number represents one of the measurement modes.

Then, the positive-sequence capacitance of three branches can be written as follows,

$$
\left\{\begin{array}{l}
C_{1}=\frac{1}{w l_{1}} \operatorname{Im}\left(\frac{1}{Y_{1}}\right) \\
C_{2}=\frac{1}{w l_{2}} \operatorname{Im}\left(\frac{1}{Y_{2}}\right) \\
C_{3}=\frac{1}{w l_{3}} \operatorname{Im}\left(\frac{1}{Y_{3}}\right)
\end{array}\right.
$$

where $l_{1}, l_{2}$ and $l_{3}$ are the length of three branches; $\mathrm{w}=2 \pi f$ and $f$ is power frequency.

\section{Digital Simulation Results}

According to the above method, the positive-sequence capacitance of T-connection transmission lines is simulated under the three cases. Each branch is applied with an external positive-sequence voltage source in turn and the other two branches are opening circuit.

The simulation results of three different cases are shown in Tables 2-4 respectively.

Table 2. The Simulation Results of Three Branches with the Same Length.

\begin{tabular}{cccc}
\hline $\begin{array}{c}\text { The length of } \\
\text { three branches } \\
(\mathrm{km})\end{array}$ & $\begin{array}{c}\text { Capacitance } \\
\text { Setting Values } \\
\text { (Unit: } \mathrm{nF} / \mathrm{km})\end{array}$ & $\begin{array}{c}\text { Capacitance } \\
\text { (Unit: } \mathrm{nF} / \mathrm{km})\end{array}$ & $\begin{array}{c}\text { Relative } \\
\text { Error } \\
(\%)\end{array}$ \\
\hline 30 & 9.15 & 9.1501 & 0.0011 \\
50 & 12.74 & 12.734 & 0.0471 \\
\hline
\end{tabular}

Table 3. The Simulation Results of Three Branches with Different Length.

\begin{tabular}{cccc}
\hline $\begin{array}{c}\text { Length } \\
(\mathrm{km})\end{array}$ & $\begin{array}{c}\text { Capacitance } \\
\text { Setting Values } \\
(\text { Unit: nF/km) }\end{array}$ & $\begin{array}{c}\text { Capacitance } \\
\text { Mearement Values } \\
(\text { Unit: nF/km) }\end{array}$ & $\begin{array}{c}\text { Relative } \\
\text { Error } \\
(\%)\end{array}$ \\
\hline Branch 1: 10 & 12.74 & 12.830 & 0.71 \\
Branch 2: 20 & 9.15 & 9.3793 & 2.51 \\
Branch 3: 30 & 14.00 & 13.816 & -1.31 \\
\hline
\end{tabular}

Table 4. The Simulation Results of Three Branches with Different Length.

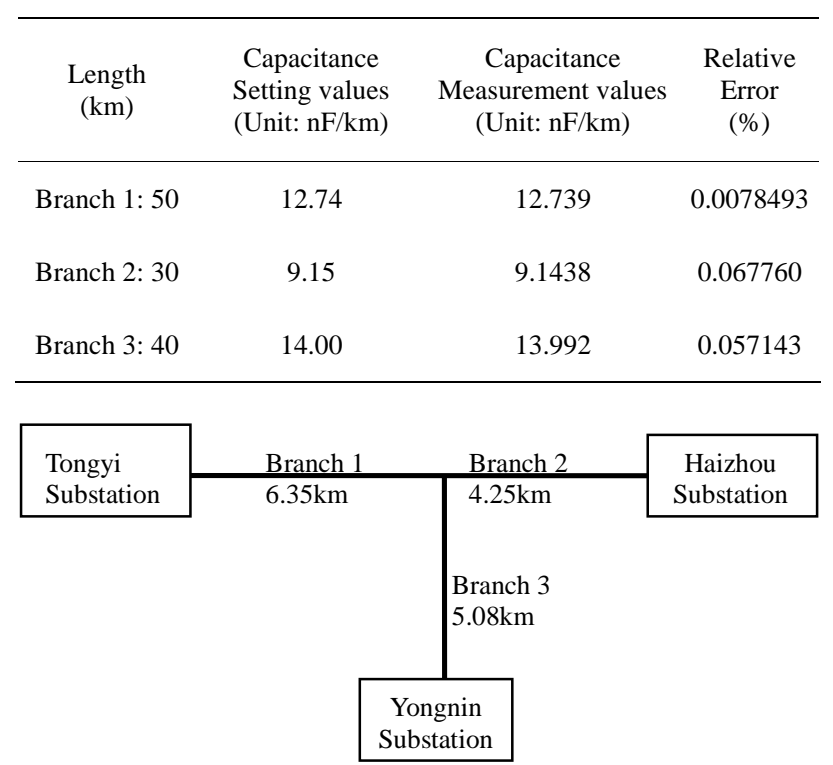

Figure 3. Diagram of the T-connection transmission lines.

Table 5. The Positive-Sequence Capacitance Measurement Results of Three Branches.

\begin{tabular}{|c|c|c|c|}
\hline $\begin{array}{l}\text { Length } \\
(\mathrm{km})\end{array}$ & $\begin{array}{c}\text { Capacitance } \\
\text { Calculation Values } \\
\text { (Unit: } \mathrm{nF} \text { ) }\end{array}$ & $\begin{array}{c}\text { Capacitance } \\
\text { Measurement Values } \\
\text { (Unit: } n F)\end{array}$ & $\begin{array}{l}\text { Relative } \\
\text { Error } \\
(\%)\end{array}$ \\
\hline Branch 1: 6.35 km & 58.075 & & \\
\hline Branch 2: $4.25 \mathrm{~km}$ & 38.869 & & \\
\hline Branch 3: 5.08 km & 46.459 & & \\
\hline
\end{tabular}

\section{An Example of Field Test}

The new measurement method has been successfully used in measuring the positive-sequence capacitance parameters of $110 \mathrm{kV}$ T-connection transmission lines in a power grid as shown in Figure 3.

The independent measuring cases of the T-connection line are shown in Table 1.

The positive-sequence capacitance measurement results with the new method are shown in Table 5.

\section{Conclusions}

The new measurement method of the positive-sequence capacitance parameters of $\mathrm{T}$-connection transmission lines is introduced. The theoretical analysis, digital simulation results and the field measurement results have proven that the new measurement method is correct and can be used for measuring the positive-sequence capacitance parameters of T-connection transmission lines. 


\section{Acknowledgements}

This work was financially supported by the Ph.D. Programs Foundation of Ministry of Education of China (20110141110032).

\section{REFERENCES}

[1] Z. Hu and Y. Chen, "New Method of Live Line Measuring the Inductance Parameters of Transmission Lines Based on GPS Technology," IEEE Transactions on Power Delivery, Vol. 23, No. 3, 2008, pp. 1288-2195. doi:10.1109/TPWRD.2007.916235

[2] J. R. Carson, "Wave Propagation in Overhead Wires with Ground Return,” Bell Syst. Tech. J., Vol. 5, 1926, pp. 539-556.

[3] X. Xiong, C. Zhang and Z. Hu, "Live Line Measurement to the Zero Sequence Impedance Parameters of Multi-terminal Transmission Lines,” Power System Protection and Control, Vol. 38, No. 15, 2010, pp. 65-68,
2010.

[4] L. Fang and Z. Hu. "New Method of Live Line Measuring the Parameters of T-connection Transmission Lines with Mutual Inductance and Its Engineering Application,"Power System Technology, Vol. 34, No. 4, 2010, pp. 204-208.

[5] Z. Hu. J. Fan, M. Chen and Z. Xu, "Live Line Measurement to the Inductance Parameters of T-connection Transmission Lines with Mutual Inductance," Proceedings of the IEEE PES GM 2009, July 26-30, 2009, Calgary, Alberta, Canada.

[6] J. Suonan, J. Gu, X. Xue, D. Liu and Z. Jiao, "Distance Protection Based on Frequency-domain Compensation Algorithm,” Automation of Electric Power Systems, Vol. 31, No. 23, 2007, pp. 57-60, 68.

[7] Z. Liang, B. Li, F. Lu, P. Li and Z. Yang, "Susceptance Measurement Method at Header Point of Transmission Line,” Journal of North China Electric Power University, Vol. 28, No. 1, 2001, pp. 6-9. 